\title{
Thymic carcinoma with extrinsic occlusion of the left anterior descending artery: a distinctive case of myocardial infarction in a young woman
}

\author{
Ahmed Ayuna ${ }^{1 *}$ D, Saad Ahmad', Sjirjel Alam² and Nik Abidin ${ }^{1}$
}

\begin{abstract}
Background: Thymic epithelial tumour (TET) is the most common tumour affecting the anterior mediastinum in adults. The cardiac extension is often limited to the pericardium, and intracardiac extension is rare. We present a unique case of encasement and displacement of the left anterior descending coronary artery by the large mediastinal tumour leading to myocardial ischemia.

Case presentation: Our patient is a 28-year-old lady with stage 4 TET. She presented with acute chest pain associated with 12-lead ECG changes and a significant rise in serial troponin I. Multimodality cardiac imaging revealed encasement and displacement of the left anterior descending coronary artery by the large mediastinal tumour. CT-FFR demonstrates evidence of ischemia which would account for her acute presentation. Following detailed MDT discussions between cardiologists, oncologists and cardiothoracic surgeons, the decision was made to treat this lady with palliative chemotherapy. Given the extent of the tumour invasion and failure of the initial therapy, her prognosis and the outcome were poor.

Conclusions: TET could cause atrial compression, myocardial infiltration, and invasion of the pulmonary and caval veins; however, to the best of our knowledge, this is the first case reported of coronary artery displacement and encasement by TET.
\end{abstract}

Keywords: Thymoma, Thymic carcinoma, TET, Coronary artery displacement, Myocardial ischemia, CT-FFR

\section{Background}

Thymic epithelial tumour (TET) is the most common tumour affecting the anterior mediastinum in adults [1]. However, they are still relatively rare tumours attributing from 0.2 to $1.5 \%$ of all malignancies [2]. TET most commonly affects adults who are between 40 and 60 years old, and the incidence is similar between males and females [3]. The cardiac extension is often limited to the pericardium, and intracardiac extension is rare [4]. Moreover, TET could be associated with distant metastasis to the liver, skin and lung (0-10\%, mean 5.5\%) [5].

\footnotetext{
* Correspondence: drahmedayuna@gmail.com

'Salford Royal NHS Foundation Trust, Manchester, UK

Full list of author information is available at the end of the article
}

In general, cardiac involvement in cancer patients can cause atrial compression, myocardial infiltration and invasion of the pulmonary and caval veins [6]. In thymic cancer, it has been reported to involve the right and left atrium, left ventricle and pulmonary veins [4]; however, to the best of our knowledge, this is the first case reported of coronary artery displacement and encasement by TET.

Nevertheless, coronary artery compression by a metastatic cardiac tumour resulting in acute coronary syndrome has been reported previously, and it was associated with poor outcome [7]. Thomas and colleagues have reported a case series of five cases of malignant thymoma with intracardiac extensions [4]. The 


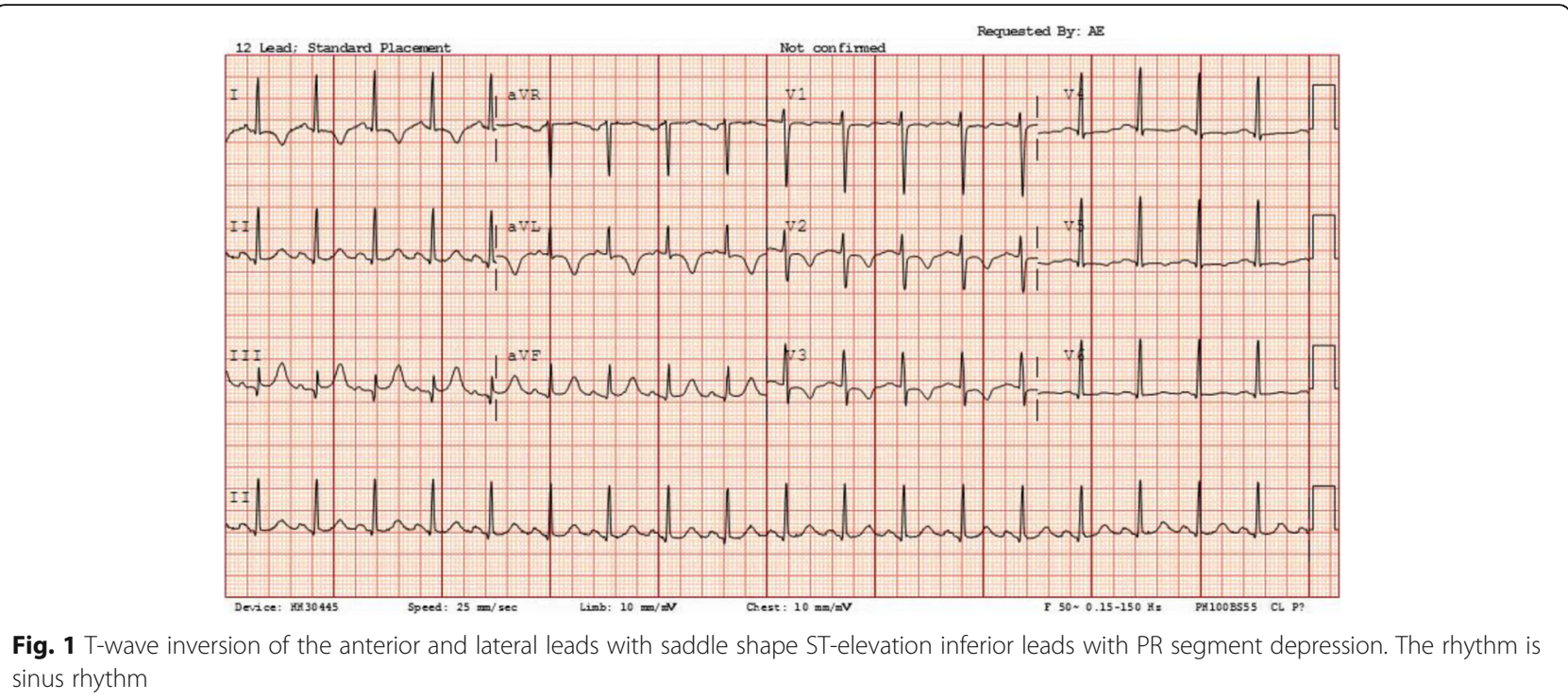

cardiac extension involved the left ventricular outflow and caused haemodynamic compromise. Guidance for the definitive management of thymoma and thymic carcinomas is still premature due to the lack of randomised trials $[8,9]$. Unfortunately, the majority of the patients with thymic carcinomas present with advanced-stage tumour [4]. The prognosis is mainly influenced by the stage and the complete resectability of the tumour, i.e. the higher the stage, the worse the prognosis [10]. In our case report, the patient was consented and all the data used was anonymised.

\section{Case presentation}

A 28-year-old lady with metastatic thymic epithelial tumour (TET) presented to our hospital with worsening anginal chest pain. She was post-partum, having delivered a healthy baby girl just 7 weeks earlier. She has known malignancy with stage 4 thymic cancer, which has spread to the pleura and lymph nodes at the time of diagnosis and was managed with chemotherapy. Her initial diagnosis was made 4 months ago and confirmed with the tissue sample histopathology, which revealed an epithelial cancer of the thymus. The initial diagnosis was made when she was 6 months pregnant and developed rapidly progressive shortness of breath.

Although her presentation this time was non-pleuritic chest pain, given her pre-existing co-morbidities, the main differential upon first assessment was that of pulmonary embolism. She had a raised D-dimer, and the fact that she has malignancy can increase the risk of thromboembolism. ECG, however, appeared ischaemic with ST depression and T-wave inversion across V1-V3 and in leads I and aVL (Fig. 1). Given this lady's young age and lack of cardiovascular risk factors, acute coronary syndrome (ACS) was thought to be unlikely.

CT pulmonary angiogram (CTPA) was performed and demonstrated no evidence of pulmonary embolism (PE); however, it showed the already known mediastinal mass.
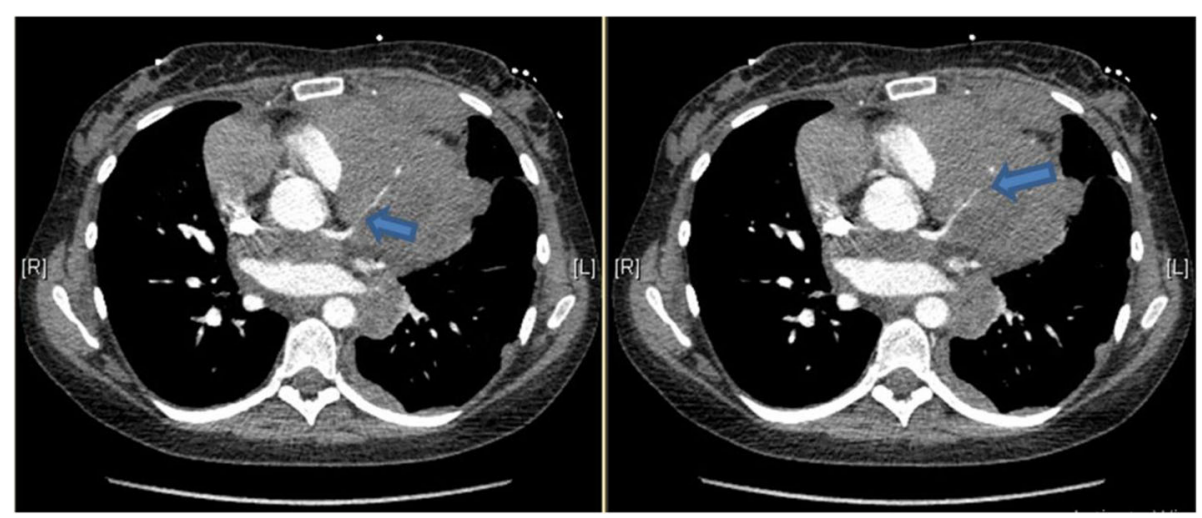

Fig. 2 CT pulmonary angiogram reveals encasement and displacement of the left anterior descending coronary artery (blue arrows) 


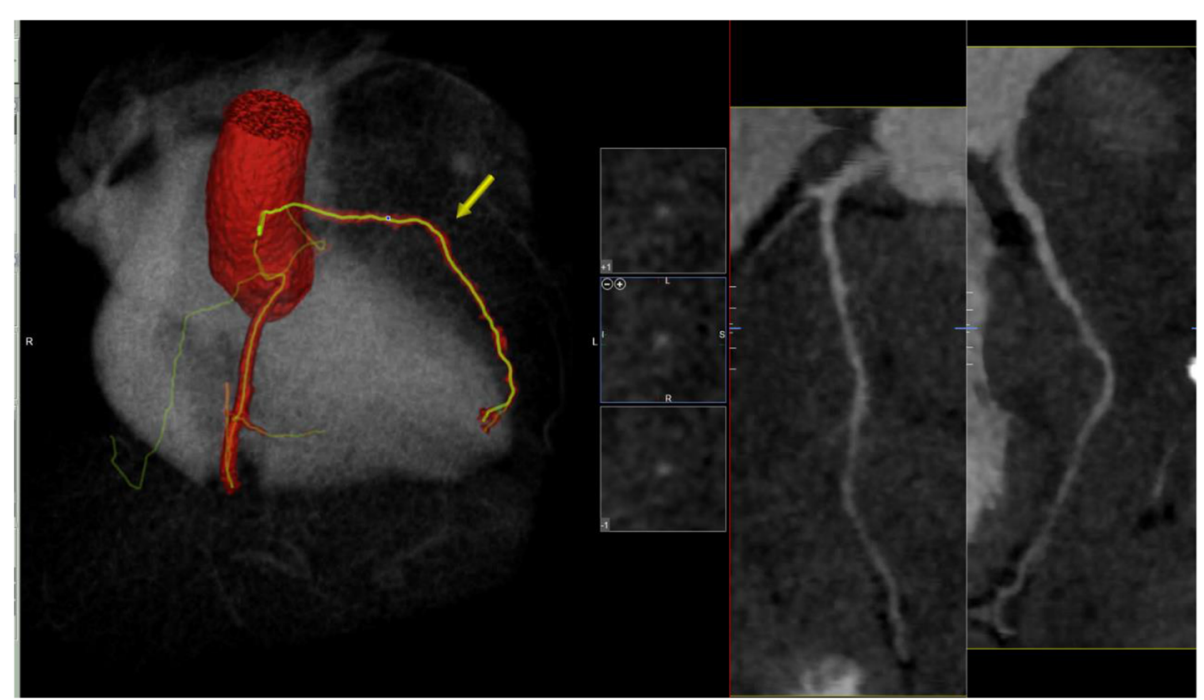

Fig. 3 CT coronary angiogram reveals displacement of the left anterior descending coronary artery (LAD) yellow arrow. Right anterior oblique-cranial projection

The cancer appeared to have increased in size compared with her previous CT images, and it has encased the great vessels. There was compression of the pulmonary trunk and both pulmonary arteries from a large $130 \times$ $92 \times 109 \mathrm{~mm}$ tumour. There was the involvement of the pleura and pericardium and, most notably, encasement and displacement of the left anterior descending artery (LAD) (Fig. 2). CT coronary angiogram (CTCA) showed displacement of the LAD with a significant reduction in the CT-FFR distal vessel indicating flow-limiting tumour compression (Figs. 3, 4, 5).
She was diagnosed with acute coronary syndrome supported by serial Troponin I levels of $223 \mathrm{ng} / \mathrm{L}$ and 628 ng/L. Echocardiography showed a mass compressing the right ventricular outflow tract (RVOT) (Fig. 6) and it confirmed mild to moderate LV systolic dysfunction $(45 \%+/-5$ LV ejection fraction by Simpson's Biplane method) and anterolateral hypokinesia (Supplementary movie). A unifying diagnosis of LAD compression secondary to cancerous mass effect was apparent. She was commenced on dual antiplatelet therapy, glyceryl trinitrate $(\mathrm{GTN})$ infusion for ongoing angina pains and

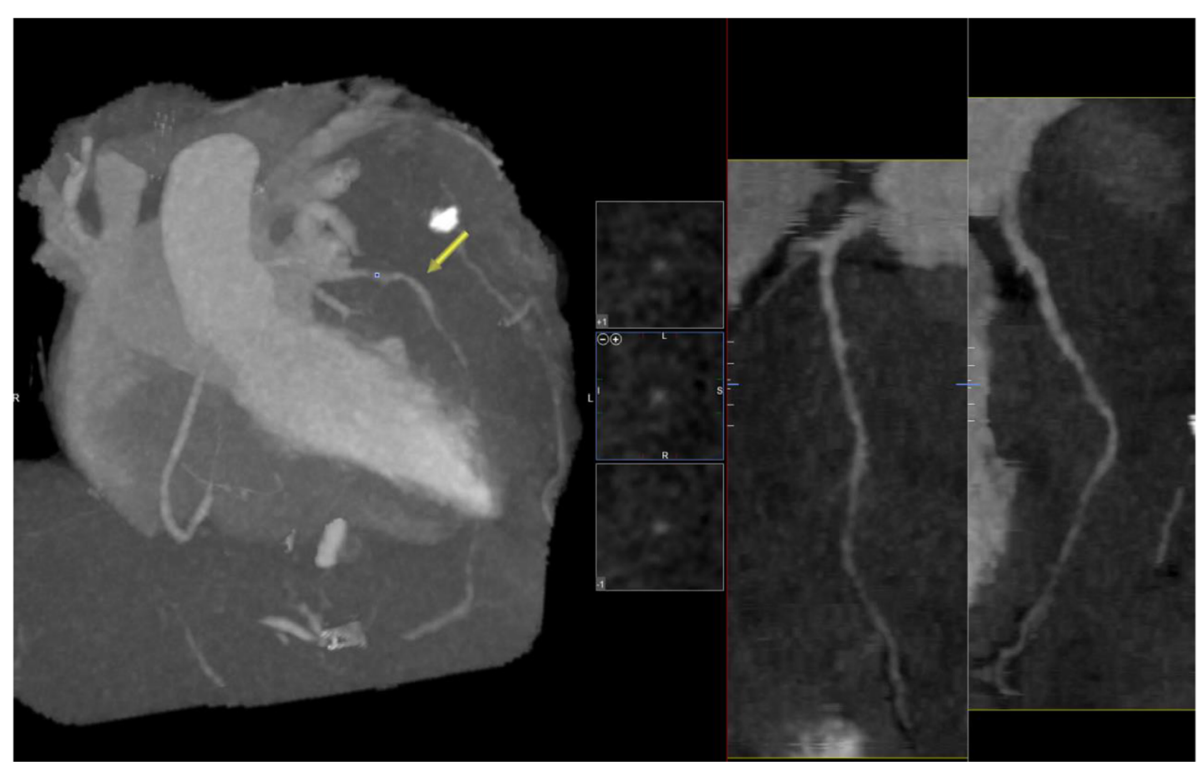

Fig. $4 \mathrm{CT}$ coronary angiogram reveals displacement of the left anterior descending coronary artery (LAD) yellow arrow. Right anterior oblique projection 


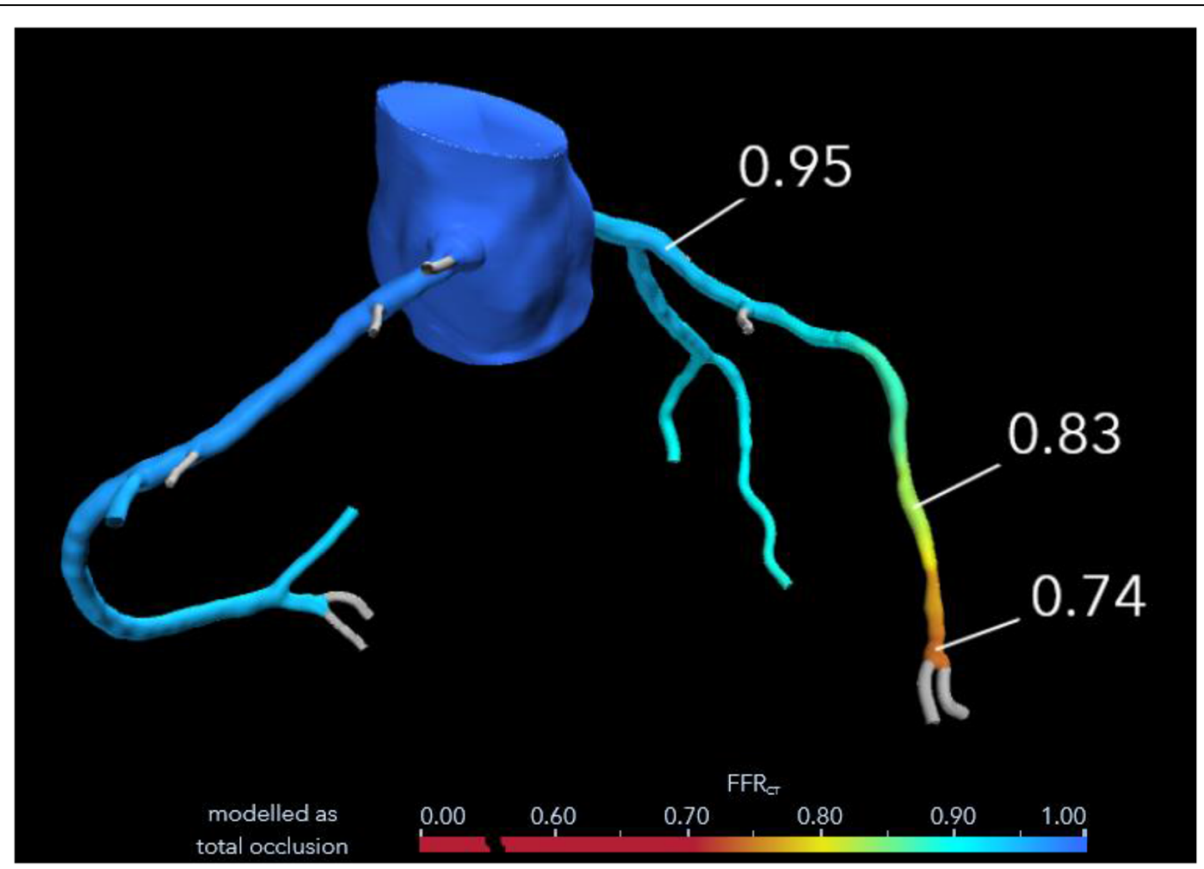

Fig. 5 CT coronary angiogram with CT-FFR indicates distal left anterior descending coronary artery (LAD) ischemia. Right anterior oblique-cranial projection

subsequently transferred urgently to the local tertiary cardiac centre for further management.

Her case was reviewed and discussed at the multidisciplinary team (MDT) meeting. The consideration was for her to proceed directly to coronary angiography with a view to percutaneous coronary intervention (PCI). PCI and stenting for extrinsic coronary artery compression is an unusual undertaking with a variable success rate in the context of extrinsic tumour displacement and compression $[11,12]$. However, PCI has been used to relieve

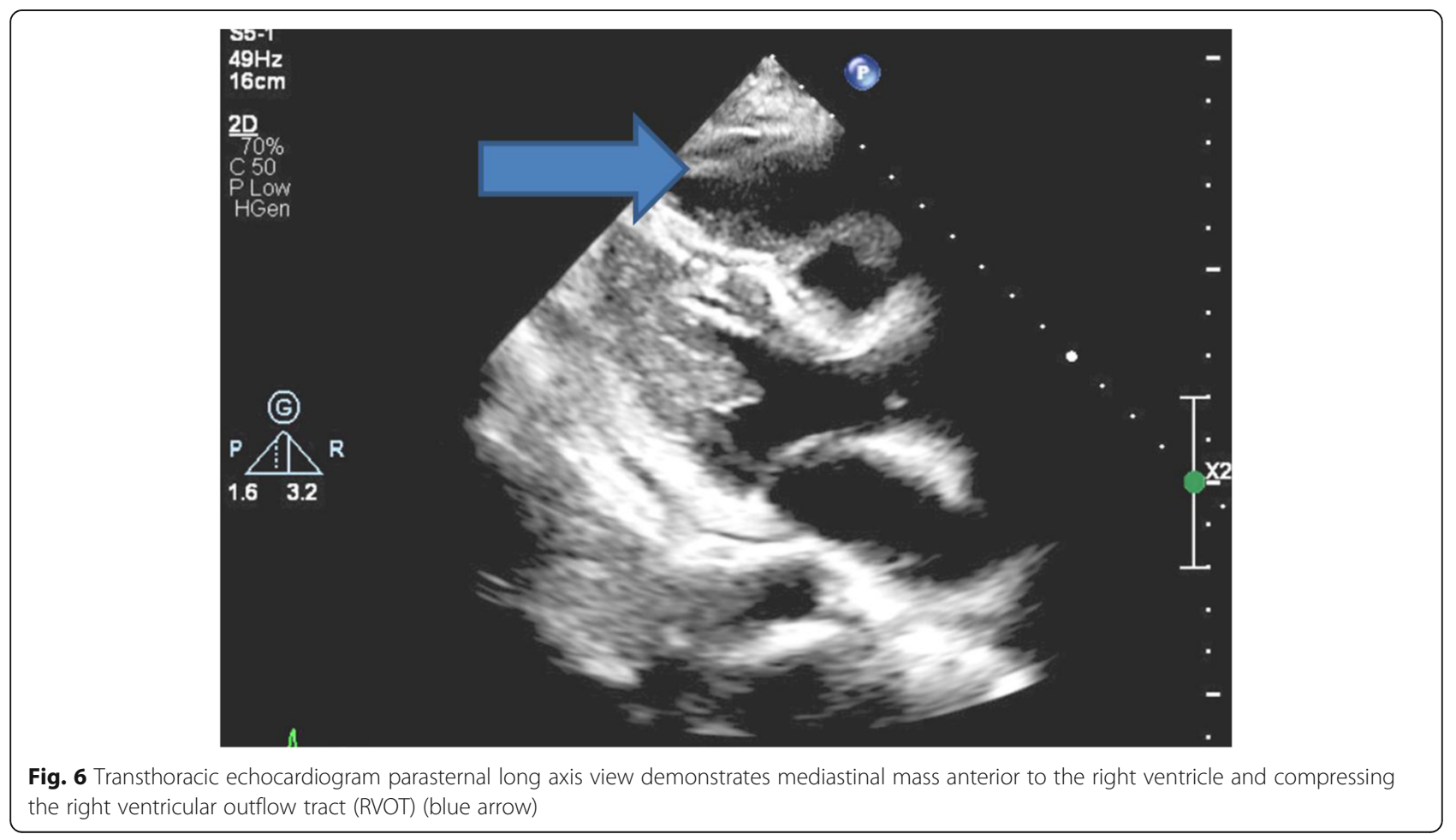


coronary artery compression by other tumours with a guarded prognosis [7]; we could not find any previous report to use the PCI in the context of compressive TET. Following detailed MDT discussions between cardiologists and oncologists, the decision was made to treat this lady with palliative chemotherapy. Given the extent of the tumour invasion and failure of the initial therapy, her prognosis and outcome were poor, and the patient has been referred to the palliative care team for symptoms control.

\section{Conclusions}

TET is a common mediastinal tumour, but cardiac involvement is rare. To the best of our knowledge, this is the first case report of a patient with TET presenting with cardiac chest pain as a consequence of encasement and displacement of the coronary artery by an enlarging TET. This case highlights the importance of cardiac and chest imaging in making the diagnosis and guiding treatment options and follow-up care.

\section{Abbreviations}

ACS: Acute coronary syndrome; ECG: Electrocardiogram; CTCA: Computed tomography coronary angiography; CT-FFR: Computed tomography fractional flow reserve; CTPA: Computed tomography pulmonary angiography; MDT: Multi-disciplinary team; PE: Pulmonary embolism; RVOT: Right ventricular outflow tract; TET: Thymic epithelial tumour

\section{Supplementary Information}

The online version contains supplementary material available at https://doi. org/10.1186/s43044-021-00178-1.

Additional file 1. Movie of transthoracic echocardiogram parasternal short axis view demonstrates regional wall motion abnormalitieshypokinesia of the anterolateral segment.

\section{Acknowledgements}

Not applicable

\section{Authors' contributions}

The authors have read and approved the final manuscript. AA - conception and design, drafting the chapter, data collection; SAH - drafting the chapter, data collection; SA - conception and design, revising the chapter critically for important intellectual content; NA - conception and design, revising the chapter critically for important intellectual content.

\section{Funding}

This work was not supported by any fund.

Availability of data and materials

Not applicable

\section{Declarations}

Ethics approval and consent to participate

Not applicable

Consent for publication

The authors have a written consent from the patient.

Competing interests

The authors declare that they have no competing interests.

\section{Author details}

${ }^{1}$ Salford Royal NHS Foundation Trust, Manchester, UK. ${ }^{2}$ Manchester

Universities Foundation Trust, Manchester, UK.

Received: 15 March 2021 Accepted: 5 June 2021

Published online: 02 July 2021

\section{References}

1. Engles EA, Pfeiffer RM (2003) Malignant thymoma in the United States: demographic patterns in incidence and associations with subsequent malignancies. In J Cancer 105:546-551

2. Fornasiero A, Daniele O, Ghiotto C, Sartori F, Rea F, Piazza M, Fiore-Donati L, Morandi P, Aversa SM, Paccagnella A (1990) Chemotherapy of invasive thymoma. J Clin Oncol 8(8):1419-1423. https://doi.org/10.1200/JCO.1990.8. 8.1419

3. Sadieddine N, Liu G, Cuningham K et al (2014) Prognostic factors for cure, recurrence and long-term survival after surgical resection of thymoma. J Thorac Oncol 9(7):1018-1022. https://doi.org/10.1097/JTO.000000000000021 5

4. Thomas A, Shanbhag S, Haglund K, Berman A, Jakopovic M, Szabo E, Arai A, Schrump DS, Kwong KF, Rajan A, Giaccone G (2013) Characterization and management of cardiac involvement of thymic epithelial tumours. J Thorac Oncol 8(2):246-249. https://doi.org/10.1097/JTO.0b013e31827bd931

5. Kondo K, Monden Y (2003) Lymphogenous and haematogenous metastasis of thymic epithelial tumours. Ann Thorac Surg 76(6):1859-1864. https://doi. org/10.1016/S0003-4975(03)01017-8

6. Chiles C, Woodard PK, Gutierrez FR, Link KM (2001) Metastatic involvement of the heart and pericardium: CT and MR imaging. Radiographics 21(2):439449. https://doi.org/10.1148/radiographics.21.2.g01mr15439

7. Ozaki T, Chiba S, Annen K, Kawamukai Y, Kohno N, Horimoto M (2009) Acute coronary syndrome due to coronary artery compression by a metastatic cardiac tumor. J Cardiol Cases 10:e52-e55

8. Falkson CB, Bezjak A, Darling G, Gregg R, Malthaner R, Maziak DE, Yu E, Smith CA, McNair S, Ung YC, Evans WK, Lung Cancer Disease Site Group of Cancer Care Ontario's Program in Evidence-Based Care (2009) The management of thymoma: a systematic review and practice guideline. J Thorac Oncol 4(7):911-919. https://doi.org/10.1097/JTO.0b013e3181a4b8e0

9. PDQ Adult Treatment Editorial Board (2020) Thymoma and thymic carcinoma treatment (adult) (PDQ $\left.{ }^{\oplus}\right)$ : health professional version. In: PDQ Cancer Information Summaries [Internet]. National Cancer Institute (US), Bethesda (MD) 2002-2020 Oct 2. Available online at https://pubmed.ncbi. nlm.nih.gov/26389476/ (accessed 03/05/2021)

10. Ströbel P, Bauer A, Puppe B, Kraushaar T, Krein A, Toyka K, Gold R, Semik M, Kiefer R, Nix W, Schalke B, Müller-Hermelink HK, Marx A (2004) Tumour recurrence and survival in patients treated for thymomas and thymic carcinoma squamous cell carcinomas: a retrospective analysis. J Clin Oncol 22(8):1501-1509. https://doi.org/10.1200/JCO.2004.10.113

11. Batra K, Saboo SS, Kandathil A, Canan A, Hedgire SS, Chamarthy MR, Kalva SP, Abbara S (2020) Extrinsic compression of coronary and pulmonary vasculature. Cardiovasc Diagnosis Therapy Available online at 10.21037/cdt20-155 (accessed 23/05/2021)

12. Ferlini M, Dore R, lannopollo G, De Ferrari GM, Oltrona Visconti L (2018) Left main coronary artery external compression by lung adenocarcinoma successfully treated with percutaneous coronary intervention. J Cardiovasc Med 19(11):674-676. https://doi.org/10.2459/JCM.0000000000000715

\section{Publisher's Note}

Springer Nature remains neutral with regard to jurisdictional claims in published maps and institutional affiliations. 